EGU21-6320

https://doi.org/10.5194/egusphere-egu21-6320

EGU General Assembly 2021

(c) Author(s) 2021. This work is distributed under

the Creative Commons Attribution 4.0 License.

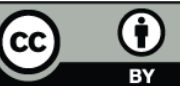

\title{
What is the most threatening for citizens of a mid-latitude city: cold stress or heat stress?
}

\author{
Sara Top ${ }^{1,2}$, Dragan Milošević ${ }^{3}$, Steven Caluwaerts ${ }^{1,4}$, and Stevan Savić ${ }^{3}$ \\ ${ }^{1}$ Department of Physics and Astronomy, Ghent University, Ghent, Belgium (sara.top@ugent.be) \\ ${ }^{2}$ Department of Geography, Ghent University, Ghent, Belgium \\ ${ }^{3}$ Climatology and Hydrology Research Centre, University of Novi Sad, Novi Sad, Serbia \\ ${ }^{4}$ Royal Meteorological Institute of Belgium, Brussels, Belgium
}

Both heat and cold waves cause extreme human thermal discomfort and a clear excess in mortality. This shows the importance of knowing the prevailing thermal comfort conditions and how thermal comfort conditions vary in various environments so measures can be taken. Microclimatic and outdoor human thermal comfort conditions are investigated in various built-up and green areas in the city of Ghent (Belgium) using meteorological measurements of six weather stations of the MOCCA (Monitoring the Cities Climate and Atmosphere) network in combination with calculations done by RayMan.

Normal to extreme summer heat wave periods show that dangerous strong heat stress prevails during the daytime periods at all locations. Comparison of thermal comfort during normal and extreme summer heat wave periods showed that heat stress is more extreme when a heat wave is more intense. Overall the urban park in Ghent was the most comfortable location during heat waves since it effectively mitigates heat stress in the city. These results should be taken into account in urban planning and design to keep mid-latitude cities livable.

Further, a one year data series revealed that outdoor cold stress was more apparent during 2017 in the mid-latitude city of Ghent that experiences a mild maritime climate. During spring and summer, both heat stress and cold stress occurred due to the larger diurnal temperature range compared to the other seasons. Even though high Physiological Equivalent Temperatures (PET) were obtained during a heat wave in summer, heat stress did not occur as intensely and as frequently compared to cold stress on annual level. It could thus be stated that outdoors, cold stress is a bigger threat than heat stress. However, one should keep in mind that the study was executed for outdoor thermal heat comfort and that people will take shelter or take measures when feeling uncomfortable. The question is thus rather, how are citizens protected against heat and cold stress? Currently, the Belgian society is better adapted to cold stress since most buildings contain central heating, while air conditioning is not standard. Future projections predict an increase in temperature, causing more occurrence of extreme heat stress, while extreme cold stress will be reduced. Additionally, the urban heat island effect currently has mainly a positive effect on the average annual outdoor thermal comfort conditions, while it will become a negative 
effect in the warmer future. Measures should thus be taken to reduce the threat of future heat stress. 